\title{
Value of multiplex PCR for detection of antimicrobial resistance in samples retrieved from patients with orthopaedic infections
}

Irene Katharina Sigmund ${ }^{1,2+}$, Nora Renz ${ }^{1+}$, Susanne Feihl ${ }^{1}$, Christian Morgenstern ${ }^{1}$, Sabrina Cabric ${ }^{1,2}$ and Andrej Trampuz ${ }^{1,3^{*}}$

\begin{abstract}
Background: The performance of multiplex PCR (mPCR) for detection of antimicrobial resistance from clinical isolates is unknown. We assessed the ability of $\mathrm{mPCR}$ to analyse resistance genes directly from clinical samples. Patients with orthopedic infections were prospectively included. Phenotypical and genotypical resistance was evaluated in clinical samples (synovial and sonication fluid) where identical pathogens were identified by culture and $\mathrm{mPCR}$.

Result: A total of 94 samples were analysed, including 60 sonication fluid and 34 synovial fluid samples. For coagulase-negative staphylococcus strains, mPCR detected resistance to oxacillin in 10 of 23 isolates (44\%) and to rifampin in none of 6 isolates. For S. aureus isolates, detection rate of oxacillin and rifampin-resistance was 100\% (2/ 2 and 1/1, respectively). Fluoroquinolone-resistance was confirmed by $\mathrm{MPCR}$ in all 3 isolates of Enterobacteriaceae, in enterococci resistance to aminoglycoside-high level was detected in 1 of 3 isolates (33\%) and in streptococci resistance to macrolides/lincosamides in none of 2 isolates. The overall sensitivity for different pathogens and antimicrobials was $46 \%$ and specificity $95 \%$, the median concordance was $80 \%$ (range, 57-100\%). Full agreement was observed for oxacillin in S. aureus, vancomycin in enterococci, carbapenems/cephalosporins in Enterobacteriaceae and rifampin in Cutibacterium species.

Conclusion: The overall sensitivity for detection of antimicrobial resistance by MPCR directly from clinical samples was low. False-negative MPCR results occurred mainly in coagulase-negative staphylococci, especially for oxacillin and rifampin. However, the specificity of $\mathrm{MPCR}$ was high and a positive result reliably predicted antimicrobial resistance. Including universal primers in the PCR test assay may improve the detection rate but requires additional sequencing step.

(Continued on next page)
\end{abstract}

\footnotetext{
* Correspondence: andrej.trampuz@charite.de

${ }^{\dagger}$ Irene Katharina Sigmund and Nora Renz contributed equally to this work.

${ }^{1}$ Charité - Universitätsmedizin Berlin, corporate member of Freie Universität Berlin, Humboldt-Universität zu Berlin, and Berlin Institute of Health, Center for Musculoskeletal Surgery (CMSC), Charitéplatz 1, 10117 Berlin, Germany ${ }^{3}$ Berlin Institute of Health Center for Regenerative Therapies (BCRT), Föhrer Strasse 15, 13353 Berlin, Germany

Full list of author information is available at the end of the article
}

(c) The Author(s). 2020 Open Access This article is licensed under a Creative Commons Attribution 4.0 International License, which permits use, sharing, adaptation, distribution and reproduction in any medium or format, as long as you give appropriate credit to the original author(s) and the source, provide a link to the Creative Commons licence, and indicate if changes were made. The images or other third party material in this article are included in the article's Creative Commons licence, unless indicated otherwise in a credit line to the material. If material is not included in the article's Creative Commons licence and your intended use is not permitted by statutory regulation or exceeds the permitted use, you will need to obtain permission directly from the copyright holder. To view a copy of this licence, visit http://creativecommons.org/licenses/by/4.0/. The Creative Commons Public Domain Dedication waiver (http://creativecommons.org/publicdomain/zero/1.0/) applies to the data made available in this article, unless otherwise stated in a credit line to the data. 
(Continued from previous page)

Trial registration: www.clinicaltrials.gov No. NCT02530229, registered at 21 August 2015 (retrospectively registered).

Keywords: Multiplex PCR, Periprosthetic joint infection, Antimicrobial resistance, Sonication, Molecular methods, Diagnosis

\section{Background}

An interdisciplinary approach including surgical and antimicrobial treatment is crucial to eradicate bone and joint infection, including periprosthetic joint infections (PJI) and infections after internal bone fixation [1]. Treatment strategies are guided by the type of causative pathogen and its antimicrobial susceptibility, with special focus on biofilm-active antibiotics [2].

Previous studies demonstrated limited sensitivity of periprosthetic tissue culture $(45-71 \%)$ and synovial fluid culture (52-64\%) for microbial detection [3-8]. Cultures remain negative in particular in low- burden, chronic low-grade infections or in case of preceding antimicrobial therapy $[9,10]$. Moreover, phenotypical antimicrobial testing using conventional cultures requires several days in mixed infections and if slow-growing microorganisms are involved. Therefore, new techniques were developed to improve the diagnostic yield, such as sonication of retrieved implants and broad-range PCR of sonication fluid, improving sensitivities to $73-89 \%[3,4,10$, 11]. In a further study, inoculation of sonication fluid into blood culture bottles further improved the diagnosis of orthopaedic implant-associated infections and reduced the time to culture positivity [12].

The value of multiplex PCR (mPCR) in synovial fluid, periprosthetic tissue and sonication fluid has been recently extensively investigated [6,13-18]. Advantages of the $\mathrm{mPCR}$ technique are rapid identification of the causative pathogen and potentially detection of genetic markers for clinically relevant antimicrobial resistances [18]. However, limited data exists on the performance of mPCR for the detection of antimicrobial resistance markers in the clinical setting.

In this study, we assessed the value of a commercial mPCR assay (Unyvero i60 ITI) in genotypical detection of antimicrobial resistance, considering conventional culture the gold standard. In addition, we evaluated the concordance of susceptibility test results deriving from MPCR and culture in pathogens isolated from synovial and sonication fluid.

\section{Results}

\section{Patient demographics}

Ninety-four samples of 82 patients with a median age of 75 years (range, 28-98 years) were analysed, 47 patients $(57 \%)$ were female. The 60 sonication fluid samples originated from 38 infected prostheses (20 knee, 15 hip, 2 shoulder, and 1 elbow prosthesis) and 22 osteosyntheses (localized in the spine in 9 patients, tibia in 6, ankle in 2, femur in 2, humerus in 1 , clavicle in 1 , and elbow in 1 patient). The 34 synovial fluids were harvested from 31 infected prosthetic (13 knees and 18 hips) and 3 native septic joints (two knee and one hip joint).

\section{Microbiological characteristics}

The detected pathogens in sonication and synovial fluid samples are summarized in Table 1 . The most frequently isolated pathogens were Staphylococcus spp. (52\%), followed by Enterobacteriaceae (15\%) and Streptococcus agalactiae (12\%). Eighty-five monomicrobial and 9 polymicrobial infections were observed. In 12 patients, both sonication and synovial fluid were analysed and yielded the identical pathogen.

\section{Performance of resistance gene detection and its concordance with phenotypical resistance testing}

The detection rate of vancomycin resistance by $\mathrm{MPCR}$ in Enterococcus spp. could not be assessed as no resistance was detected by conventional culture. The same applies for Enterobacteriaceae and aminoglycosides, carbapenems, third-generation cephalosporins, as well as for Cutibacterium spp. and rifampin.

Performance of mPCR and concordance of phenotypical and genotypical susceptibility testing is summarized in Table 2 (Additional file 1). Overall, a median sensitivity of $46 \%$ and specificity of $95 \%$ was shown, with a median concordance of $80 \%$. There was no difference seen regarding number of discordant pairs comparing synovial and sonication fluid.

\section{Staphylococcus spp}

Among Staphylococcus spp. isolates, concordance of phenotypical and genotypical susceptibility to oxacillin, aminoglycosides, macrolides/lincosamides and rifampin was $72,73,73$ and $84 \%$, respectively. The mPCR detected oxacillin resistance genes in 10 of 23 samples associated with oxacillin-resistant coagulase-negative staphylococci positive cultures (44\%), whereas both oxacillin-resistant $S$. aureus strains detected by culture were found to be resistant by mPCR (sensitivity 100\%). In coagulase-negative staphylococci, none of the rifampin resistance determined by culture was detected by $\mathrm{mPCR}$; in $S$. aureus isolates, one true positive and one false negative rifampin 
Table 1 Distribution of detected pathogens

\begin{tabular}{|c|c|c|c|}
\hline Microbiology & Sonication fluid $(\boldsymbol{n}=60)$ & Synovial fluid $(\boldsymbol{n}=34)$ & Total $(\boldsymbol{n}=94)$ \\
\hline Coagulase-negative staphylococci & 21 & 12 & $33(35 \%)$ \\
\hline Staphylococcus aureus & 9 & 7 & $16(17 \%)$ \\
\hline Streptococcus agalactiae & 4 & 7 & $11(12 \%)$ \\
\hline Enterococcus spp. ${ }^{a}$ & 8 & 2 & $10(11 \%)$ \\
\hline Escherichia coli & 4 & 5 & $9(10 \%)$ \\
\hline Cutibacterium spp. ${ }^{b}$ & 8 & 1 & $9(10 \%)$ \\
\hline Enterobacter cloacae & 3 & 0 & $3(3 \%)$ \\
\hline Finegoldia magna & 1 & 0 & $1(1 \%)$ \\
\hline Proteus spp. & 1 & 0 & $1(1 \%)$ \\
\hline Klebsiella pneumoniae & 1 & 0 & $1(1 \%)$ \\
\hline
\end{tabular}

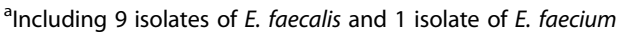

${ }^{b}$ Including 8 isolates of $C$. acnes and 1 isolate of $C$. avidum

resistance was detected by $\mathrm{mPCR}$, resulting in a NPV of $100 \%$ and a PPV of $50 \%$.

\section{Enterococcus spp}

The vancomycin antimicrobial resistance testing showed $100 \%$ agreement in 10 isolates (all tested susceptible). Among 7 tested strains, one isolate was tested resistant to aminoglycosides with both methods. Genotypical high-level resistance to aminoglycosides was determined in 1 sample, whereas phenotypical resistance was determined by culture in 3 isolates. Thus, MPCR missed the resistance to aminoglycosides detected through aac $\left(6^{\prime}\right)$ / $\operatorname{aph}\left(2^{\prime \prime}\right)$ in 2 samples (66\%). The resulting concordance was $71 \%$ with a sensitivity, specificity, NPV and PPV of $33,100,67$ and 100\%, respectively.

\section{Enterobacteriaceae}

The overall agreement for the testing of different antimicrobial agents in 14 Enterobacteriaceae isolates was good with mPCR test results showing NPV of $100 \%$ for all antimicrobial agents. $\mathrm{mPCR}$ detected resistance to fluoroquinolones in all 3 isolates. False-positive resistance was detected for aminoglycosides in 4 patients, resulting in a PPV of $0 \%$ and for fluoroquinolones in 1 patient with a PPV of $75 \%$.

\section{Streptococcus spp}

Of 10 Streptococcus agalactiae isolates, lincosamides by culture/lincosamides by culture and ten by mPCR, resulting in a concordance and NPV of $80 \%$ and a specificity of $100 \%$. As no resistant strain was detected, PPV could not be assessed.

\section{Anaerobes}

For rifampin, all 6 isolates of Cutibacterium spp. showed concordance (susceptibility by culture and mPCR). In one sonication fluid sample, Finegoldia magna was identified by culture and mPCR. This pathogen was susceptible to amoxicillin/clavulanic acid, penicillin, piperacillin/tazobactam, imipenem, clindamycin, rifampin and levofloxacin by conventional susceptibility testing using culture. However, no markers are available for resistance testing for this pathogen using the mPCR system.

\section{Discussion}

Rapid identification of a pathogen and its susceptibility pattern allows effective optimization of the antimicrobial treatment and prevention of emergence of resistance. While conventional culture is the gold-standard microbiological method, molecular methods are increasingly used in the clinical routine. However, limitations of molecular detection of drug-resistance genes need to be considered, in particular the fact that genotypic drug-resistance identification does not conclusively corresponds to the phenotype [19]. In addition, focusing on the detection of a certain selection of genes with $\mathrm{mPCR}$, the diversity of all bacterial resistance genes is not represented.

The sensitivity and specificity of $\mathrm{MPCR}$ for pathogen detection directly from clinical samples found in the published sub-cohorts ranged in sonication fluid from 51 to $71 \%$ and 92 to $94 \%$, respectively, and in synovial fluid from 23 to $60 \%$ and 89 to $91 \%$, respectively [6-8, 17]. Previous publications using $\mathrm{mPCR}$ for pathogen identification reported similar sensitivities ranging from 51 to $96 \%$ and specificities from 94 to $100 \%[13,16,18$, $20,21]$. However, limited data exists on the performance of the MPCR system for detection of resistance genes and their agreement on conventional antimicrobial susceptibility testing in patients with bone and joint infections [18, 22].

A recent study [23] employing the first generation of the multiplex PCR device reported a low detection rate (6\%) of $m e c A$ and $m e c C$ genes in methicillin-resistant staphylococci (not specified which subspecies), which 


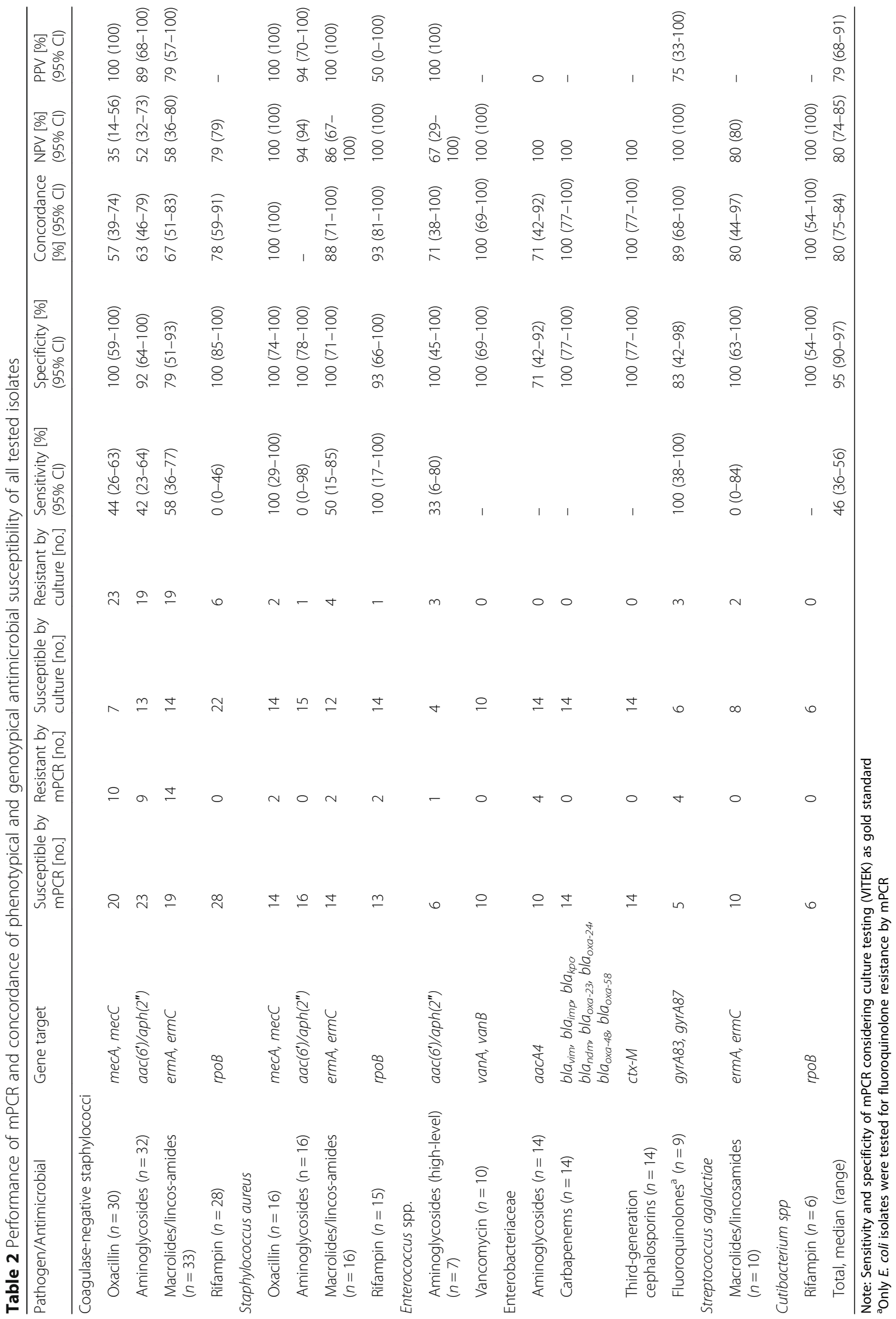


increased to $35 \%$ when using the second generation of the mPCR cartridge. In our cohort, we observed a detection rate of $48 \%$ (12 of 25 isolates) for oxacillin resistance. However, the oxacillin-resistance detection rate in $S$. aureus was considerably higher than in coagulasenegative staphylococci (100\% versus $44 \%)$. The limited detection of oxacillin resistance in coagulase-negative staphylococci by mPCR may be explained by the low microbial burden usually seen in low grade infections caused by less virulent staphylococci, which probably does not reach the detection limit of the MPCR system of mecA and $m e c C$, estimated at $\sim 10^{4}$ and $\sim 10^{6}$ DNA copies $/ \mathrm{ml}$, respectively. This hypothesis is corroborated by the fact, that the concordance in infections caused by S. aureus - usually acute infections - was considerably higher. Furthermore, a great diversity of the staphylococcal cassette chromosome mec in coagulase-negative staphylococci may contribute to a reduced detection rate of oxacillin resistance [24].

From the clinical viewpoint, susceptibility to rifampin is of paramount relevance in implant-associated infections caused by staphylococci and Cutibacterium spp., as this antibiotic is the only biofilm-active antibiotic for these microorganisms $[25,26]$. Pre-operative knowledge of rifampin resistance influences the surgical decision, as a lack of biofilm-active treatment usually discourages retention of the implant in acute infections and a one-stage exchange in chronic infections [1]. In our cohort, the concordance between genotypical and phenotypical evaluation for rifampin was moderate for Staphylococcus spp. (84\%) with $86 \%$ false-negative test-result (i.e. phenotypically resistant and genotypically susceptible) and excellent for Cutibacterium spp. (100\%). However, the cartridge is not validated for rifampin susceptibility testing in Cutibacterium spp. and coagulase-negative staphylococci. When analysing $S$. aureus only, for which the system is validated, a concordance of $93 \%$ with a negative predictive value of $100 \%$ was obtained. Similarly, fluoroquinolones represent the biofilm-active agent for implant-associated infections caused by gram-negative bacteria [27]. For all analysed $E$. coli strains, a concordance of $89 \%$ for fluoroquinolones with a $100 \%$ NPV was detected.

With 71 to $73 \%$, the concordance of phenotypical and genotypical testing for aminoglycosides was in the lower range for all tested pathogens, i.e. staphylococci, enterococci and gram-negative rods. For enterococci and streptococci, the NPV was low due to missed resistance by $\mathrm{mPCR}$. In contrast, in gram-negative rods falsepositive MPCR test resulted in low PPV (0\%).

We acknowledge several limitations of this study. First, given the low number of rifampin-resistant staphylococci and fluoroquinolone-resistant Enterobacteriaceae, its value for detection of pathogens causing difficult-totreat infections is limited and their predictive values from our study should be taken with caution. Additionally, we could not assess detection rates for several resistance genes, as we did not include any isolates tested resistant by conventional culture (e.g. gram-negative bacilli resistant to aminoglycosides, third generation cephalosporines and carbapenems). Another limitation represents the inclusion of exclusively those clinical samples, which were concordant in pathogen detection by culture and $\mathrm{MPCR}$. The performance of $\mathrm{MPCR}$ in discordant samples is unknown and the specificity results of this study may be overestimated. Furthermore, we have not investigated the performance of $\mathrm{MPCR}$ in viable but non culturable (VBNC) condition, where the number of DNA copies may be much lower than that in growing condition. Finally, an analysis between the quantitative MIC values (i.e., strength of resistance) and detectability of resistance genes (i.e., numbers of DNA copies) was not performed in our study, which might provide additional insights in the performance of mPCR for detection of antimicrobial resistance.

\section{Conclusion}

The overall sensitivity for detection of antimicrobial resistance by mPCR directly from clinical samples was low. Due to low sensitivity the evaluated commercial mPCR system cannot replace conventional antimicrobial resistance testing. False-negative $\mathrm{mPCR}$ results occurred mainly in coagulase-negative staphylococci, especially for oxacillin and rifampin. However, the specificity of mPCR was high and a positive result reliably predicts resistance and for some pathogens and antibiotics full agreement was observed, including for oxacillin in S. aureus, vancomycin in enterococci, carbapenems/cephalosporins in Enterobacteriaceae and rifampin in Cutibacterium species. Including universal primers in the PCR test assay may improve the detection rate but requires additional sequencing step.

\section{Methods \\ Study design}

This prospective single-center study (public clinical trial identification at www.clinicaltrials.gov NCT02530229) was conducted in accordance with the Declaration of Helsinki. Approval of the institutional review board was obtained (EA1/306/14) and patients provided written informed consent before study inclusion. The results of mPCR were not used to guide antimicrobial therapy. Data analysed in this study represent a subpopulation of previously published cohorts analysing the performance of mPCR with regards to detection of bacteria in synovial fluid of native joints [7] and periprosthetic joints [17], as well as in sonication fluid from removed joint prostheses [6] and other orthopaedic hardware [8]. Among 378 samples (179 sonication fluids [128 
prostheses, 51 osteosyntheses], 199 synovial fluids [142 prosthetic joints, 57 native joints]) enrolled in the abovementioned published cohort studies, 94 samples detected the same microorganisms using $\mathrm{MPCR}$ and conventional culture. Two-hundred-nine specimens with no microbial growth, 73 patients with discordant microorganisms and two cases, where no phenotypical drug resistance analysis was performed, were excluded. A study selection flow diagram is shown in Fig. 1.

\section{Study population}

Consecutive adult patients who had undergone diagnostic joint aspiration for suspected septic arthritis or revision surgery for implant-associated bone infection between November 2014 and October 2015 were included. Four patients in whom sonication fluid of retrieved osteosynthesis was investigated, were receiving antimicrobial treatment at time of explantation. No other patients had been pre-treated with antibiotics.

\section{Joint aspiration}

Synovial fluid was aspirated by an orthopaedic surgeon according to standardized aseptic technique. After skin preparation, synovial fluid was collected using a sterile 18 -gauge spinal needle and a $10-\mathrm{ml}$ syringe. If no synovial fluid was obtained, the needle was repositioned without withdrawing it through the skin.

\section{Synovial fluid cultures}

0.1 milliliter of synovial fluid was inoculated onto aerobic and anaerobic plates (sheep blood agar, chocolate agar, and Schädler anaerobic agar), and $1 \mathrm{ml}$ was inoculated in thioglycolate broth. Agar plates were incubated at $37^{\circ} \mathrm{C}$ under aerobic and anaerobic conditions for 14 days and inspected daily for microbial growth. Microbial identification was performed using Vitek2 (bioMérieux, Nürtingen, Germany) or a matrix-associated laser desorption/ionization-time of flight mass spectrometer (bioMérieux). The antimicrobial susceptibility testing was conducted using Vitek 2 and the minimum inhibitory concentration (MIC) values were determined and interpreted according to EUCAST standards. For mPCR analysis, $2-5 \mathrm{ml}$ of the synovial or $10-50 \mathrm{ml}$ of sonication fluid was transferred to a sterile vial and stored at $-80^{\circ} \mathrm{C}$ until processing.

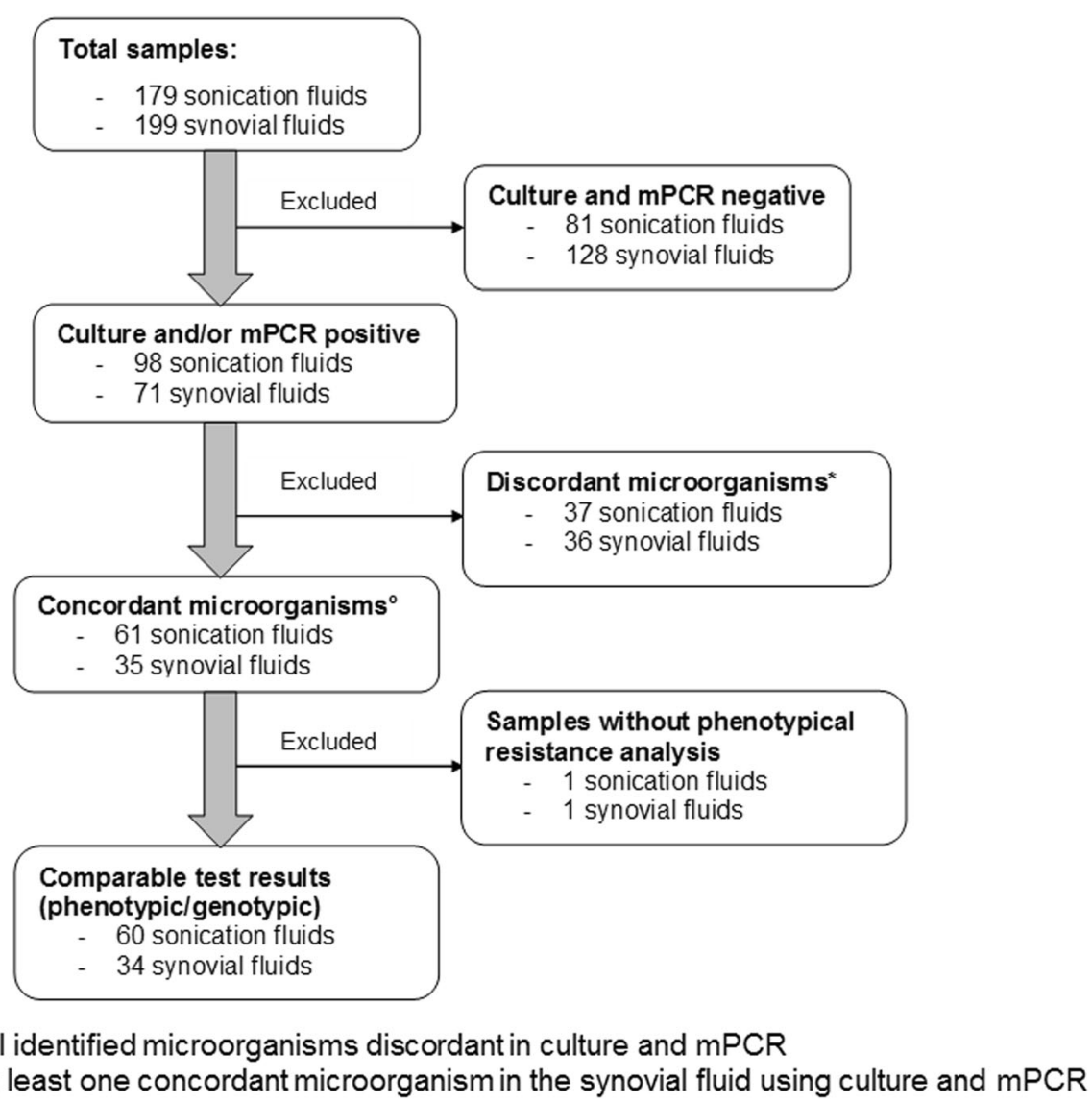

Fig. 1 Study selection flow diagram 


\section{Removal and transport of orthopedic hardware}

The joint prosthesis components (metal, ceramic or polyethylene parts) or other fracture fixation devices (plates, nails or screws) were removed in the operating room. After removal, the hardware components were transported to the microbiological laboratory in a sterile air-tight container (Lock\&Lock, Frankfurt am Main, Germany) and processed within 6 hours.

\section{Sonication procedure}

In the microbiology laboratory, normal saline was added to the container, covering at least $80 \%$ of the hardware. The container was vortexed for $30 \mathrm{~s}$ and sonicated for 1 minute at $40 \mathrm{kHz}$ and $0.2 \mathrm{~W} / \mathrm{cm}^{2}$ (BactoSonic, Bandelin electronic, Berlin, Germany). Additional vortexing was conducted for $30 \mathrm{~s}$ before the sonication fluid was plated. Then, $0.1 \mathrm{ml}$ of the sonication fluid was inoculated onto agar plates and $1 \mathrm{ml}$ was inoculated in thioglycolate broth. Microorganisms were enumerated (i.e. number of colony-forming unit $[\mathrm{CFU}] / \mathrm{ml}$ sonication fluid). Microbial identification and antimicrobial susceptibility testing were performed as described above for synovial fluid.

\section{Multiplex PCR analysis}

The Unyvero i60 ITI application (Unyvero i60, Curetis, Holzgerlingen, Germany, second generation), designed for a semi-quantitative DNA determination parallelly performing eight multiplex PCR reactions, was used. This instrument is capable to detect 114 nucleic acids and 20 drug resistance markers. Fifty milliliter of the sonication fluid were first centrifuged at $4000 \mathrm{x}$ g at $4{ }^{\circ} \mathrm{C}$ for $20 \mathrm{~min}$ (Microcentrifuge 5427R, Eppendorf, Wesseling-Berzdorf, Germany). The supernatant was discarded and $180 \mu \mathrm{l}$ of the pellet were further processed. The synovial fluid was processed directly without centrifugation. The Unyvero i60 ITI application was used according to the manufacturer's protocol. In brief, the pre-treated sample was treated with proteinase $\mathrm{K}$ for $30 \mathrm{~min}$, master mix was added and inserted into the Unyvero ITI Cartridge. The cartridge device is equipped with eight separate chambers, a corresponding number of arrays, reagent containers and a DNA purification column. The assembled and closed Unyvero ITI Cartridge was inserted into the Unyvero Analyzer, which then automatically processed the sample within 5 hours. The microbial identification and antibiotic resistance markers are displayed on the screen of the instrument. A sample was considered positive if at least one of the analytes (pathogens) reached the threshold of $10^{4}$ DNA copies/ml.

According to the manufacturer, the resistance markers aacA4, ctx-M, ermA, gyrA83, mecA, vanA and the betalactamase genes $b l a_{n d m}, b a_{\text {oxa }-23}$, bla $_{\text {oxa }-48}$, bla $_{\text {oxa-58 }}$ were detected at a concentration of $10^{4}$ DNA copies $/ \mathrm{ml}$; the
Table 3 Resistance markers detected by the multiplex PCR test panel

\begin{tabular}{|c|c|}
\hline Antibiotics & Resistance markers \\
\hline Oxacillin & mecA, mecC \\
\hline Aminoglycosides & $\operatorname{aac}\left(6^{\prime}\right) / \operatorname{aph}\left(2^{\prime \prime}\right), \operatorname{aacA4}$ \\
\hline Macrolides/lincosamides & ermA, ermC \\
\hline Vancomycin & $\operatorname{van} A, \operatorname{van} B$ \\
\hline Rifampin ${ }^{a}$ & $r p o B$ \\
\hline Third-generation cephalosporins & $c t x-M$ \\
\hline Carbapenems & $\begin{array}{l}\text { bla } a_{\text {vim, }} \text {, bla } a_{i m p}, \text { bla } a_{k p c} \text {, bla } a_{n d m}, \text { bla } a_{\text {oxa-23, }} \\
\text { bla }_{\text {oxa-24, }} \text { bla oxa-48, bla oxa-58 }\end{array}$ \\
\hline Fluoroquinolones $^{\mathrm{b}}$ & gyrA83, gyrA87 \\
\hline
\end{tabular}

${ }^{a}$ Rifampin resistance testing is only validated for Staphylococcus aureus ${ }^{\mathrm{b}}$ Fluoroquinolone resistance testing is only validated for Escherichia coli among gram-negative bacteria

resistance markers $\operatorname{aac}\left(6^{\prime}\right) / \operatorname{aph}\left(2^{\prime \prime}\right), \quad$ gyrA87, bla imp $_{\text {, }}$ $b_{b l a_{k p o}} b l a_{o x a-24}$, and $b l a_{v i m}$ were detected at a concentration of $10^{5}$ DNA copies $/ \mathrm{ml}$; and the resistance markers erm $C$, mecC, rpoB, and $v a n B$ at a concentration of $10^{6}$ DNA copies $/ \mathrm{ml}$. The range of antibiotic resistance genes detected with the mPCR system is shown in Table 3.

\section{Statistical analysis}

Categorical variables are described as absolute frequencies and percentages. Metrical variables are expressed by median and range, as appropriate. The performance of mPCR (i.e. sensitivity and specificity) and its positive predictive value (PPV) and negative predictive value (NPV) were assessed in terms of detection of resistance, using phenotypical testing by conventional culture as gold standard. Concordance analysis was performed comparing phenotypical and genotypical resistance test results, for substances, for which genotypical resistance markers were included in the MPCR cartridge. The software packages XLSTATPM (version 2017; XLSTAT; Addinsoft, New York, NY, USA).

\section{Supplementary information}

Supplementary information accompanies this paper at https://doi.org/10. 1186/s12866-020-01741-7.

Additional file 1. Raw data of the concordant microorganisms and their detected resistances by $\mathrm{MPCR}$ and culture.

\section{Abbreviations}

CFU: Colony-forming unit; DNA: Deoxyribonucleic acid; MIC: Minimum inhibitory concentration; mPCR: Multiplex polymerase chain reaction; NPV: Negative predictive value; PJI: Periprosthetic joint infection; PPV: Positive predictive value

Acknowledgements

Not applicable. 


\section{Authors' contributions}

IKS, NR, AT: conception and design of the study. SC, IKS, CM: acquisition of data. IKS, NR, SF: analysis and interpretation of data. IKS, NR, SF, CM, SC, AT: drafting the article or revising it critically for important intellectual content. IKS, NR, SF, CM, SC, AT: final approval of the version to be submitted. The authors have read and approved the manuscript.

\section{Funding}

This work was funded by PRO-IMPLANT Foundation, Berlin, Germany (https:// www.pro-implant.org), a non-profit organization supporting research, education, global networking and care of patients with bone, joint or implantassociated infection. The study was also supported by Curetis providing diagnostic PCR test kits. The funding had no influence on the data analysis or interpretation of the results. We acknowledge also support from the German Research Foundation (DFG) and the Open Access Publication Fund of Charité - Universitätsmedizin Berlin, Germany.

\section{Availability of data and materials}

All data generated or analysed during this study are included in this published article.

\section{Ethics approval and consent to participate}

Approval of the institutional review board (Charité - Universitätsmedizin Berlin, Germany) was obtained (EA1/306/14) and patients provided written informed consent before study inclusion.

\section{Consent for publication}

Not applicable.

\section{Competing interests}

The authors declare that they have no competing interests.

\section{Author details}

${ }^{1}$ Charité - Universitätsmedizin Berlin, corporate member of Freie Universität Berlin, Humboldt-Universität zu Berlin, and Berlin Institute of Health, Center for Musculoskeletal Surgery (CMSC), Charitéplatz 1, 10117 Berlin, Germany. ${ }^{2}$ Department of Orthopaedics and Trauma Surgery, Medical University of Vienna, Spitalgasse 23, 1090 Vienna, Austria. ${ }^{3}$ Berlin Institute of Health Center for Regenerative Therapies (BCRT), Föhrer Strasse 15, 13353 Berlin, Germany.

\section{Received: 11 April 2019 Accepted: 3 March 2020}

\section{Published online: 14 April 2020}

\section{References}

1. Zimmerli W, Trampuz A, Ochsner PE. Prosthetic-joint infections. N Engl J Med. 2004;351(16):1645-54

2. Del Pozo JL, Patel R. Clinical practice. Infection associated with prosthetic joints. N Engl J Med. 2009;361(8):787-94.

3. Holinka J, Bauer L, Hirschl AM, Graninger W, Windhager R, Presterl E. Sonication cultures of explanted components as an add-on test to routinely conducted microbiological diagnostics improve pathogen detection. J Orthop Res. 2011;29(4):617-22.

4. Gomez E, Cazanave C, Cunningham SA, Greenwood-Quaintance KE, Steckelberg JM, Uhl JR, et al. Prosthetic joint infection diagnosis using broad-range PCR of biofilms dislodged from knee and hip arthroplasty surfaces using sonication. J Clin Microbiol. 2012;50(11):3501-8.

5. Atkins BL, Athanasou N, Deeks JJ, Crook DW, Simpson H, Peto TE, et al. Prospective evaluation of criteria for microbiological diagnosis of prostheticjoint infection at revision arthroplasty. The OSIRIS collaborative study group. J Clin Microbiol. 1998;36(10):2932-9.

6. Renz N, Feihl S, Cabric S, Trampuz A. Performance of automated multiplex PCR using sonication fluid for diagnosis of periprosthetic joint infection: a prospective cohort. Infection. 2017:45(6):877-84.

7. Morgenstern C, Renz N, Cabric S, Perka C, Trampuz A. Multiplex polymerase chain reaction and microcalorimetry in synovial fluid: can pathogen-based detection assays improve the diagnosis of septic arthritis? J Rheumatol. 2018:45(11):1588-93.

8. Renz N, Cabric S, Morgenstern C, Schuetz MA, Trampuz A. Value of PCR in sonication fluid for the diagnosis of orthopedic hardware-associated infections: has the molecular era arrived? Injury. 2018;49(4):806-11.
9. Berbari EF, Marculescu C, Sia I, Lahr BD, Hanssen AD, Steckelberg JM, et al. Culture-negative prosthetic joint infection. Clin Infect Dis. 2007:45(9):1113-9.

10. Trampuz A, Piper KE, Jacobson MJ, Hanssen AD, Unni KK, Osmon DR, et al. Sonication of removed hip and knee prostheses for diagnosis of infection. N Engl J Med. 2007;357(7):654-63.

11. Janz V, Wassilew Gl, Hasart O, Tohtz S, Perka C. Improvement in the detection rate of PJI in total hip arthroplasty through multiple sonicate fluid cultures. J Orthop Res. 2013;31(12):2021-4.

12. Portillo ME, Salvado M, Trampuz A, Siverio A, Alier A, Sorli L, et al. Improved diagnosis of orthopedic implant-associated infection by inoculation of sonication fluid into blood culture bottles. J Clin Microbiol. 2015;53(5):1622-7.

13. Achermann $Y$, Vogt M, Leunig M, Wust J, Trampuz A. Improved diagnosis of periprosthetic joint infection by multiplex PCR of sonication fluid from removed implants. J Clin Microbiol. 2010:48(4):1208-14.

14. Borde JP, Hacker GA, Guschl S, Serr A, Danner T, Hubner J, et al. Diagnosis of prosthetic joint infections using UMD-universal kit and the automated multiplex-PCR Unyvero i60 ITI cartridge system: a pilot study. Infection. 2015;43(5):551-60.

15. Hischebeth GT, Randau TM, Buhr JK, Wimmer MD, Hoerauf A, Molitor E, et al. Unyvero i60 implant and tissue infection (ITI) multiplex PCR system in diagnosing periprosthetic joint infection. J Microbiol Methods. 2016:121:27-32.

16. Portillo ME, Salvado M, Sorli L, Alier A, Martinez S, Trampuz A, et al. Multiplex PCR of sonication fluid accurately differentiates between prosthetic joint infection and aseptic failure. J Inf Secur. 2012;65(6):541-8.

17. Morgenstern C, Cabric S, Perka C, Trampuz A, Renz N. Synovial fluid multiplex PCR is superior to culture for detection of low-virulent pathogens causing periprosthetic joint infection. Diagn Microbiol Infect Dis. 2017;90(2): 115-9.

18. Sigmund IK, Windhager R, Sevelda F, Staats K, Puchner SE, Stenicka S, et al. Multiplex PCR Unyvero i60 ITI application improves detection of lowvirulent microorganisms in periprosthetic joint infections. Int Orthop. 2018; 43(8):1891-8.

19. Piddock $\sqcup$. Assess drug-resistance phenotypes, not just genotypes. Nat Microbiol. 2016;1(8):16120.

20. Esteban J, Alonso-Rodriguez N, del Prado G, Ortiz-Perez A, Molina-Manso D, Cordero-Ampuero J, et al. PCR-hybridization after sonication improves diagnosis of implant-related infection. Acta Orthop. 2012;83(3):299-304.

21. Melendez DP, Greenwood-Quaintance KE, Berbari EF, Osmon DR, Mandrekar $J N$, Hanssen AD, et al. Evaluation of a genus- and group-specific rapid PCR assay panel on synovial fluid for diagnosis of prosthetic knee infection. $J$ Clin Microbiol. 2016:54(1):120-6.

22. Malandain D, Bemer P, Leroy AG, Leger J, Plouzeau C, Valentin AS, et al. Assessment of the automated multiplex-PCR Unyvero i60 ITI((R)) cartridge system to diagnose prosthetic joint infection: a multicentre study. Clin Microbiol Infect. 2018;24(1):83 e1-.e6.

23. Malandain D, Bemer P, Leroy AG, Leger J, Plouzeau C, Valentin AS, et al. Assessment of the automated multiplex-PCR Unyvero i60 ITI(R) cartridge system to diagnose prosthetic joint infection: a multicentre study. Clin Microbiol Infect. 2017;24(1):83.e1-83.e6.

24. Zong Z, Peng C, Lu X. Diversity of SCCmec elements in methicillin-resistant coagulase-negative staphylococci clinical isolates. PLoS One. 2011;6(5): e20191.

25. Furustrand Tafin U, Corvec S, Betrisey B, Zimmerli W, Trampuz A. Role of rifampin against Propionibacterium acnes biofilm in vitro and in an experimental foreign-body infection model. Antimicrob Agents Chemother. 2012;56(4):1885-91.

26. Mihailescu R, Furustrand Tafin U, Corvec S, Oliva A, Betrisey B, Borens O, et al. High activity of Fosfomycin and rifampin against methicillin-resistant staphylococcus aureus biofilm in vitro and in an experimental foreign-body infection model. Antimicrob Agents Chemother. 2014;58(5):2547-53.

27. Widmer AF, Wiestner A, Frei R, Zimmerli W. Killing of nongrowing and adherent Escherichia coli determines drug efficacy in device-related infections. Antimicrob Agents Chemother. 1991;35(4):741-6.

\section{Publisher's Note}

Springer Nature remains neutral with regard to jurisdictional claims in published maps and institutional affiliations. 